\title{
Performance calculations of thermoelectric module p-type leg composed of $\left(\mathrm{Bi}_{2} \mathrm{Te}_{3}\right)_{x}\left(\mathrm{Sb}_{2} \mathrm{Te}_{3}\right)_{1-x}$
}

\author{
Michał Musiał1,2,a, Marcin Borcuch ${ }^{1,2}$ and Krzysztof Wojciechowski² \\ ${ }^{1}$ AGH University of Science and Technology, Faculty of Energy and Fuels, Department of Thermal and Fluid Flow Machines, Poland \\ ${ }^{2}$ AGH University of Science and Technology, Thermoelectric Research Laboratory, Poland
}

\begin{abstract}
The paper presents the results of numerical and simple analytical performance investigations of p-type leg of thermoelectric module (TEM). Calculations of $\left(\mathrm{Bi}_{2} \mathrm{Te}_{3}\right)_{\mathrm{x}}\left(\mathrm{Sb}_{2} \mathrm{Te}_{3}\right)_{1-\mathrm{x}}$-type leg for different chemical compositions $(\mathrm{x}=0.16,0.20,0.24,0.26)$ have been carried out to estimate the power output and conversion efficiency. Study has been performed for constant and temperature dependent thermoelectric parameters: Seebeck coefficient $\alpha$, electrical resistivity $\rho$ and thermal conductivity $k$. Results of modelling for constant material parameters fit very well with the analytical solution, pointing that numerical tools can be useful in development of thermoelectric modules and generators.
\end{abstract}

\section{Nomenclature}

$A$ Cross section area of $\mathrm{p}$-type leg $\left[\mathrm{mm}^{2}\right]$

I Current generated in the circuit $[A]$

$k \quad$ Thermal conductivity $\left[\mathrm{Wm}^{-1} \mathrm{~K}^{-1}\right]$

$L \quad$ Length of p-type leg $[\mathrm{mm}]$

$P_{L} \quad$ Power generated on the load $[\mathrm{W}]$

$\dot{Q}_{a b s}$ Heat absorbed at the hot junction $[W]$

$\dot{Q}_{C}$ Heat flux released at the cold junction $[W]$

$\dot{Q}_{H}$ Heat flux absorbed at the hot junction $[W]$

$R \quad$ Internal resistance of p-type leg $[\Omega]$

$R_{L} \quad$ Load resistance [ $\left.\Omega\right]$

$T$ Temperature $[K]$

$T_{C}$ Temperature at the cold junction $[K]$

$T_{H}$ Temperature at the hot junction $[K]$

$V_{L} \quad$ Voltage on the load $[V]$

$V_{O C}$ Open circuit voltage $[\mathrm{V}]$

$z T$ Thermoelectric figure of merit [-]

$\alpha$ Seebeck coefficient $\left[\mu V K^{1}\right]$

$\eta_{\text {mat }}$ Conversion efficiency [\%]

$\rho \quad$ Electrical resistivity $[\Omega \mathrm{mm}]$

\section{Introduction}

Thermoelectricity is a mature technology applied in large variety of fields and robustly growing year after year. As a result of Seebeck effect thermoelectric generators (TEGs) are able to directly convert heat into electricity using thermoelectric modules. Since 1960s thermoelectric generators have been used to power space probes [1] (e.g. Voyager 1 and 2) and Mars rovers (Spirit and Opportunity in 2004, Curiosity in 2011). Due to undeniable advantages such as no moving parts, no working fluid, compact design, quiet operation and no pollution generation the vast majority of these generators are still working till today. As a result of harsh EU environmental politics the issue of waste heat utilization became popular research topic worldwide, providing solutions how to improve the energy generation efficiency and limit the environment degradation problem. Solutions available on the market are expensive, complicated, requiring constant maintenance and occupying large amount of space. Thermoelectric generators since last decades have been noticed in the field of waste heat utilization. As the TEG efficiency do not satisfy the requirements to compete with other technologies, the efficiency improvements have to be done. In general TEG efficiency depends on the efficiency of thermoelectric modules and effectiveness of implemented heat exchanger [2]. Nowadays a lot of research has been carried out in the area of thermoelectric materials to develop more efficient modules to be applied in thermoelectric generators [3]. Commercially available modules with efficiency around 5\% relies on thermoelectric materials developed in 1960s [4]. State-of-the-art

\footnotetext{
$\overline{{ }^{a} \text { Corresponding author: michal@agh.edu.pl }}$
} 
solutions provide materials to construct thermoelectric modules with efficiency reaching even $15 \%$ [5]. Developed in Thermoelectric Research Laboratory segmented thermoelectric modules can operate with in higher temperatures than commercial ones with efficiency exceeding 9\% [6]. Thermoelectric materials efficiency is described using dimensionless figure of merit zT which directly corresponds with heat into electricity conversion efficiency.

$$
z T=\frac{\alpha^{2}}{\rho \cdot k} \cdot T
$$

Recent research provides materials with zT values over 1 for many materials, such as: $\mathrm{Bi}_{2} \mathrm{Te}_{3}$ alloys, $\mathrm{PbTe}$, skutterudites, SiGe etc. The highest figure of merit 2,6 has been shown for SnSe single crystals [7].

It has been proven that thermoelectric module leg (thermocouple) length ratio for maximum efficiency and power output is not the same. According to results presented in [8] maximum output power and conversion efficiency decrease with increase of the thermocouple length. Conventional thermoelectric module generates losses due to having ceramic insulator plates. Integration of TEM into heat exchangers was presented and discussed [9]. Steady-state and transient models have been prepared in order to simulate different operating conditions. Spacer-inserted thermoelectric device (SITED) has been proposed by Hoon Kim [10]. The main idea is to improve power generation and reduce thermoelectric material usage by putting low thermal conductivity material spacers. As a result of this modification electrical resistance increases, while thermal conductivity of thermoelectric modules decreases. Conclusions show the $\$ / \mathrm{W}$ ratio can be reduced by $10 \%$ sustaining $60-70 \%$ of maximum power output in contrast to maximum power output for a given zT. The importance of selecting appropriate soldering alloys was discussed in [11]. It is essential to provide stress relaxation by deformation of the solder to avoid the breakage of the legs of thermoelectric materials. The main aim of the paper is to present results of analytical and numerical investigations of simplified thermoelectric module consisting only of p-type leg. This results are first stage in further research, leading to develop numerical model of the entire thermoelectric module, taking into account both p-type and n-type materials, ceramics, connectors and thermal pastes.

\section{Simplified analytical model}

Scheme of analysed p-type leg of thermoelectric module is shown on Figure 1. Cross section area is $\mathrm{A}=72 \mathrm{~mm}^{2}$ and length is $\mathrm{L}=12 \mathrm{~mm}$. Heat is absorbed $\left(\dot{Q}_{H}\right)$ at the hot junction and liberated at the cold junction $\left(\dot{Q}_{C}\right)$. As a result of Seebeck effect, due to the temperature gradient voltage is generated. Load resistance is connected to the p-type leg and the electrical current appears in the circuit. Simple analytical performance calculations have been carried out only for constant thermoelectric properties. For temperature dependent values it is impossible to solve the equations analytically.

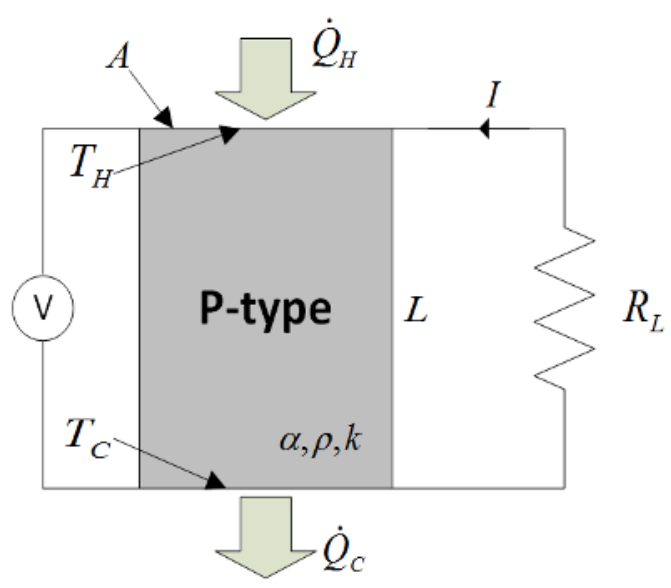

Figure 1. Scheme of p-type leg of thermoelectric module.

P-type thermocouple is made of $\left(\mathrm{Bi}_{2} \mathrm{Te}_{3}\right)_{x}\left(\mathrm{Sb}_{2} \mathrm{Te}_{3}\right)_{1-\mathrm{x}}$, material presented and discussed in [12] with different chemical compositions $(\mathrm{x}=0.26,0.24,0.20,0.16)$. Thermoelectric parameters for independent case are presented in Table 1.

Table 1. Temperature independent thermoelectric properties of p-type leg [12].

\begin{tabular}{|l|c|c|c|}
\hline $\mathrm{x}$ & $\alpha\left[\mu \mathrm{V} \cdot \mathrm{K}^{-1}\right]$ & $\rho[\Omega \cdot \mathrm{mm}]$ & $\mathrm{k}\left[\mathrm{W} \cdot \mathrm{m}^{-1} \mathrm{~K}^{-1}\right]$ \\
\hline 0.26 & 225 & 0,0168 & 1,697 \\
\hline 0.24 & 223 & 0,0147 & 1,767 \\
\hline 0.20 & 219 & 0,0134 & 1,883 \\
\hline 0.16 & 204 & 0,0101 & 1,870 \\
\hline
\end{tabular}

Parameters values for temperature dependent case are shown on figures below.

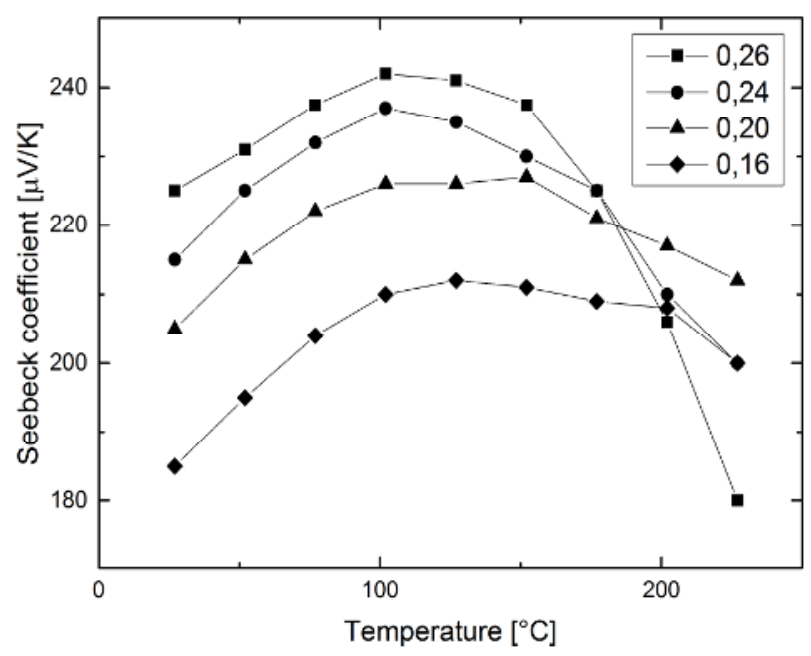

Figure 2. Temperature dependent Seebeck coefficient for different chemical compositions of p-type leg material [12]. 


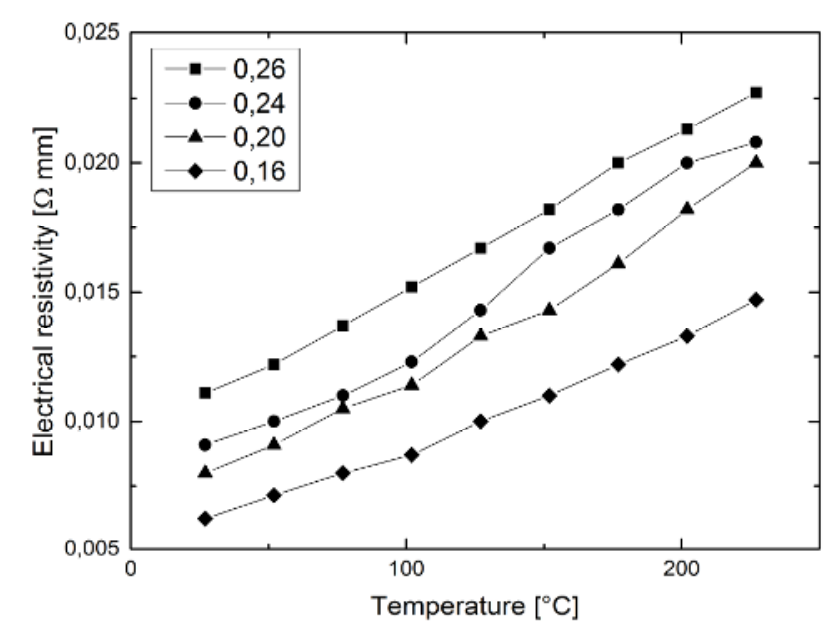

Figure 3. Temperature dependent electrical resistivity for different chemical compositions of p-type leg material [12].

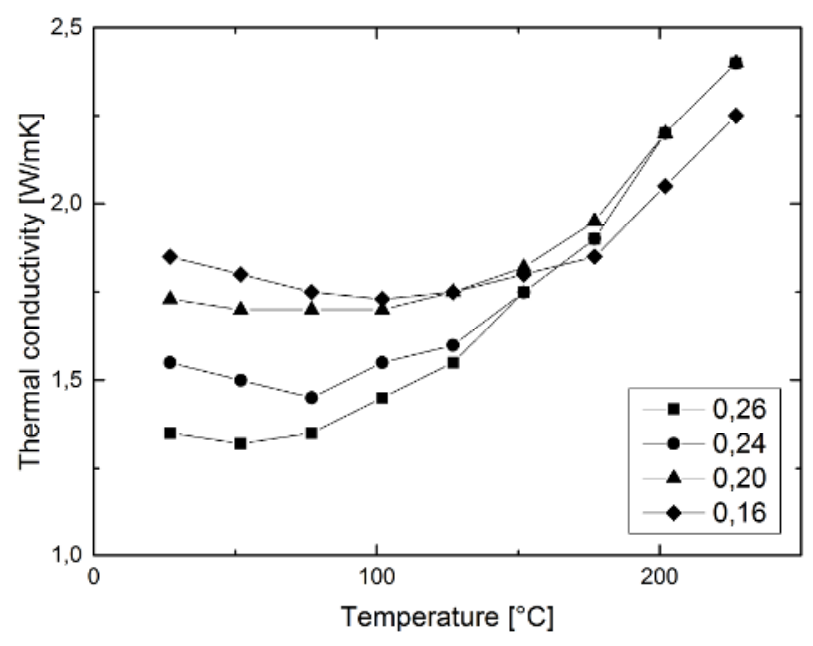

Figure 4. Temperature dependent thermal conductivity for different chemical compositions of p-type leg material [12].

Analysis has been done for hot side temperatures in the range from $27^{\circ} \mathrm{C}$ to $227^{\circ} \mathrm{C}$ with the step of $20^{\circ} \mathrm{C}$, cold side temperature remained at the level of $20^{\circ} \mathrm{C}$. As a result of Seebeck effect, due to temperature difference between hot and cold side voltage is generated. Seebeck coefficient defines amount of electrical energy generated with temperature difference of $1 \mathrm{~K}$. Voltage generated

by p-type leg can be calculated as follows:

$$
V_{O C}=\alpha \cdot\left(T_{H}-T_{C}\right) \quad[V]
$$

Internal resistance of the leg can be calculated using electrical resistivity and dimensions using equation presented below:

$$
R=\frac{\rho \cdot L}{A} \quad[\Omega]
$$

External load resistance $\mathrm{R}_{\mathrm{L}}$ was assumed to be equal to internal resistance of p-type leg, which allows to calculate performance parameters for maximum power conditions. Electrical scheme of p-type leg with load resistance connected is presented on figure 4 .

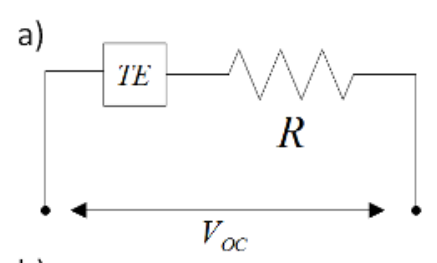

b)

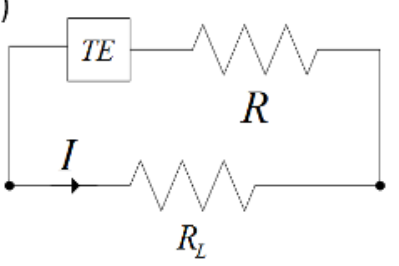

Figure 5. Electrical scheme of thermoelectric p-type leg with load resistance.

Figure 5a presents open circuit voltage, while scheme on Figure 5b represents p-type leg connected with load resistance. Current in the circuit is calculated as follows:

$$
I=\frac{V_{O C}}{R+R_{L}} \quad[A]
$$

Internal resistances for different $\mathrm{x}$ compositions of $\mathrm{p}$-type leg material are presented in Table 2.

Table 2. Temperature independent thermoelectric properties of p-type leg [12].

\begin{tabular}{|l|c|}
\hline$x$ & $R[\Omega]$ \\
\hline 0.26 & 0,00280 \\
\hline 0.24 & 0,00245 \\
\hline 0.20 & 0,00224 \\
\hline 0.16 & 0,00169 \\
\hline
\end{tabular}

Voltage across the load and maximum power generated on the load can be obtained by:

$$
\begin{aligned}
& V_{L}=I \cdot R_{L} \quad[V] \\
& P_{L}=I^{2} \cdot R_{L} \quad[W]
\end{aligned}
$$

Heat absorbed by the material at the hot junction is calculated using:

$$
Q_{a b s}=\alpha \cdot T_{H} \cdot I-\frac{1}{2} \cdot I^{2} \cdot R+\frac{k \cdot A}{L} \cdot\left(T_{H}-T_{C}\right) \quad[W]
$$

Conversion efficiency, defined as the ratio of power generated by the module and heat absorbed is as follows:

$$
\eta_{m a t}=\frac{P_{L}}{Q_{a b s}} \cdot 100 \% \quad[\%]
$$

This material efficiency do not take into account ceramics, connectors and copper base resistances. It is assumed that the efficiency of entire thermoelectric module is around one fourth of material efficiency. 


\section{Numerical model}

Steady-state numerical model has been prepared in order to simulate the thermoelectric effect in p-type leg of thermoelectric module. Fine hexahedral mesh presented on Figure 6, composed of 12000 elements with orthogonal quality equals to 1 has been developed to decrease calculation time.

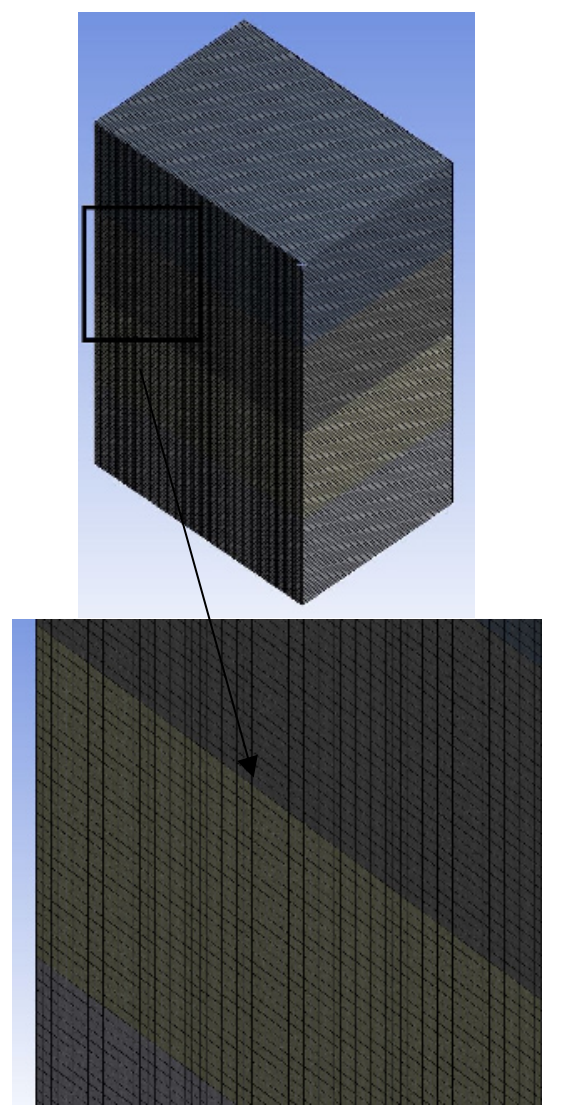

Figure 6. Hexahedral mesh developed for p-type leg of thermoelectric module.

Heat flux and electric current flows from hot side to cold side of the p-type leg. Simulation takes into account only heat transport due to Fourier's law and voltage generation as a result of Seebeck effect. Load resistance is included to assess the power generated.Thermal and electrical boundary conditions for the model have been defined as follows:

- Hot side temperature $27^{\circ} \mathrm{C}-227^{\circ} \mathrm{C}$ (with the step of $20^{\circ} \mathrm{C}$ );

- Cold side temperature remained constant: $20^{\circ} \mathrm{C}$;

- Thermal insulation condition has been defined to exclude heat transfer between p-type leg and surrounding;

- Voltage at the hot side of p-type leg has been set as $0 \mathrm{~V}$;

- Load resistance for constant $\alpha, \rho, \mathrm{k}$ has the same value as internal resistance of p-type leg simulations for maximum power generated on the load;

- Load resistance for temperature-dependent $\alpha, \rho, \mathrm{k}$ has the value equals to temperature-averaged internal resistance of p-type leg.

\section{Results}

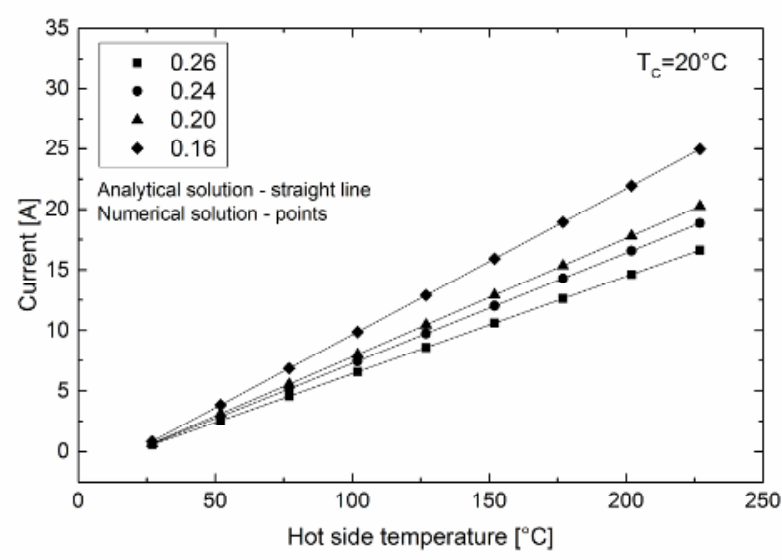

Figure 7. Results of analytical and numerical calculations of current in the function of hot side temperature for different chemical compositions and constant $\alpha, \rho, \mathrm{k}$.

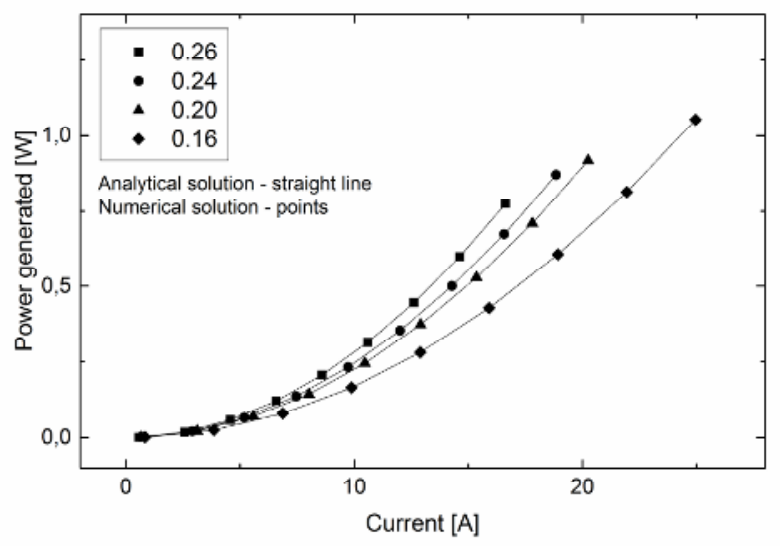

Figure 8. Results of analytical and numerical calculations of generated power in the function of current for different chemical compositions and constant $\alpha, \rho, \mathrm{k}$.

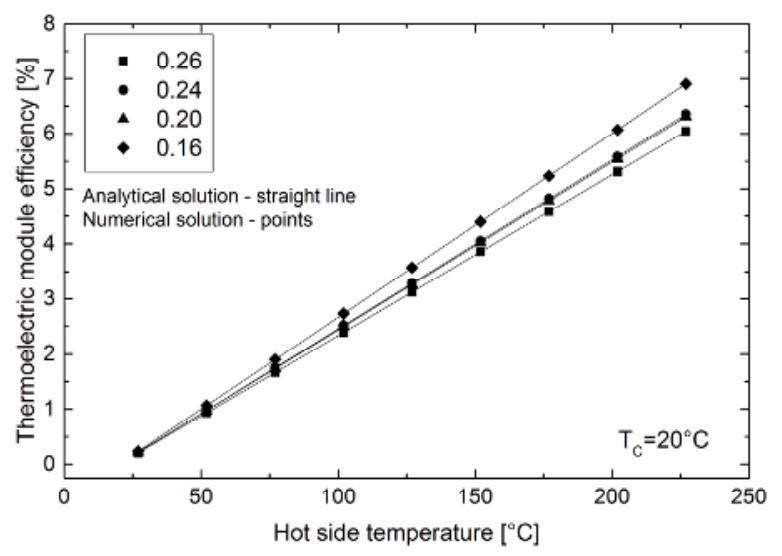

Figure 9. Results of analytical and numerical calculations of conversion efficiency in the function of hot side temperature for different chemical compositions and constant $\alpha, \rho, \mathrm{k}$.

Figures 7-9 presents the results of analytical calculations compared with numerical simulations. Maximum performance parameters are noticed for $\mathrm{x}=0.16$. This material has the highest conversion efficiency $6,9 \%$ for $\mathrm{T}_{\mathrm{H}}=227^{\circ} \mathrm{C}$. 


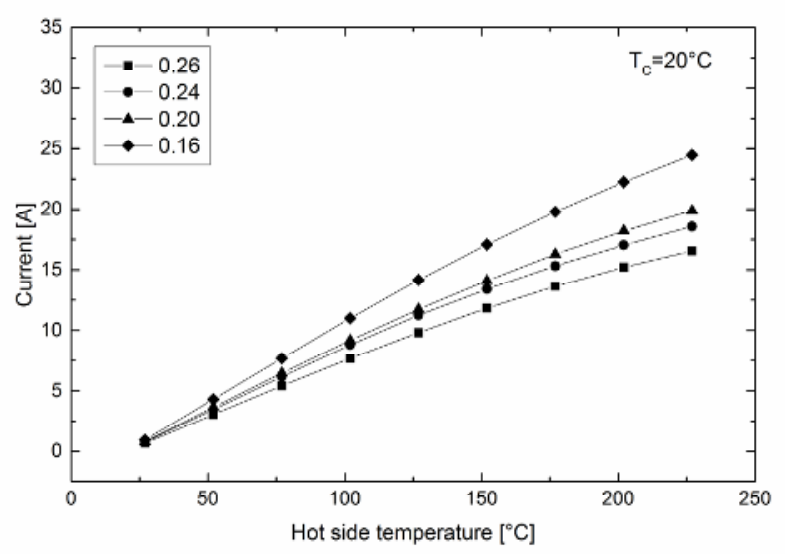

Figure 10. Results of numerical calculations of current in the function of hot side temperature for different chemical compositions and temperature dependent $\alpha, \rho, \mathrm{k}$.

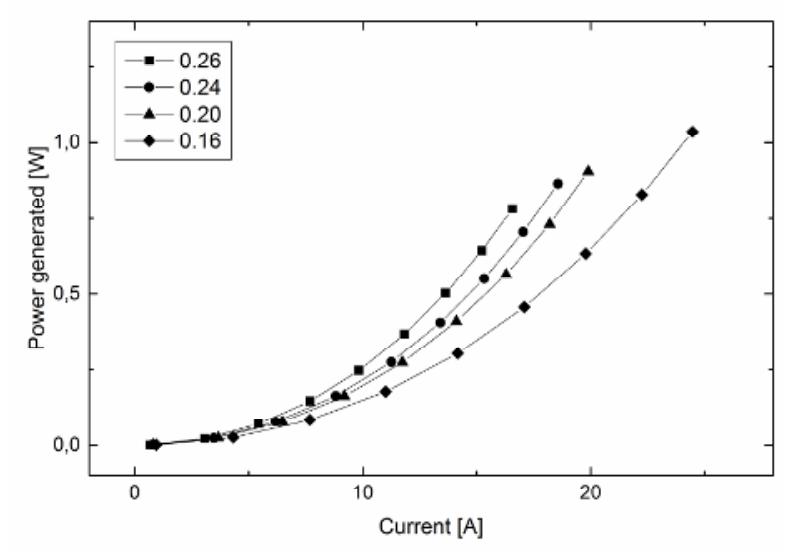

Figure 11. Results of numerical calculations of generated power in the function of current for different chemical compositions and temperature dependent $\alpha, \rho, \mathrm{k}$.

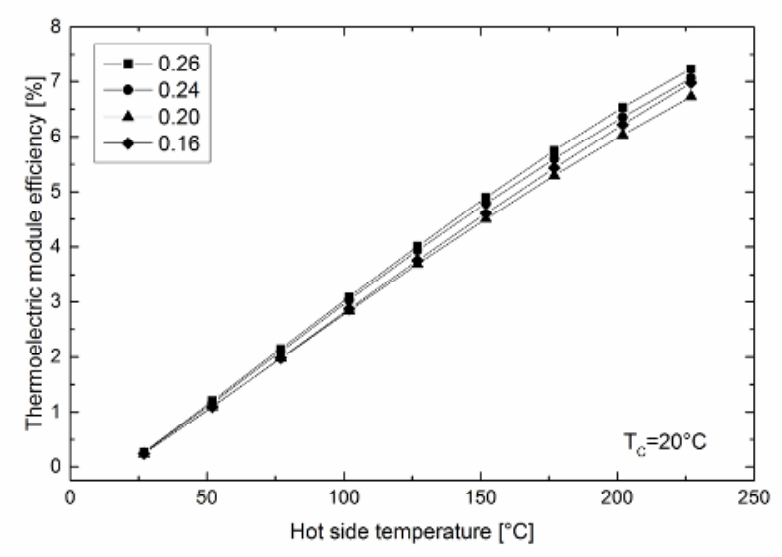

Figure 12. Results of numerical calculations of conversion efficiency in the function of hot side temperature for different chemical compositions and temperature dependent $\alpha, \rho, \mathrm{k}$.

Results of calculations and simulations for temperature dependent thermoelectric properties are shown of Figures $10-12$. In contrast to constant $\alpha, \rho, \mathrm{k}$ case performance parameters are not linear functions of temperature. For this case maximum efficiency exceeds $7,32 \%$ in $\mathrm{T}_{\mathrm{H}}=$ $227^{\circ} \mathrm{C}$ for $\mathrm{x}=0.26$.

\section{Conclusions}

The paper presents the results of performance calculations for p-type leg of thermoelectric module composed of $\left(\mathrm{Bi}_{2} \mathrm{Te}_{3}\right)_{x}\left(\mathrm{Sb}_{2} \mathrm{Te}_{3}\right)_{1-\mathrm{x}}$ p-type leg for different chemical compositions $(x=0.16,0.20,0.24,0.26)$. Simplified analytical model concerning only thermoelectric material has been developed to estimate current, power generated and conversion efficiency of $\mathrm{p}$ type leg. Two cases have been considered, the first with constant Seebeck coefficient, electrical resistivity and thermal conductivity, while second for parameters varied with temperature. Due to non-linear problems that can occur when temperature dependent thermoelectric parameters are taken into consideration the analytical calculations have been done only for the case with constant $\alpha, \rho$ and $k$. Numerical calculations have been carried out for both cases. Numerical model results have been confirmed with simple algebraic calculations. Current generated in the circuit is clearly the highest for $\mathrm{x}=0.16$. For temperature independent part relation between current and hot side temperature is linear, while for temperature dependent case is not. The most power is generated on the load for $\mathrm{x}=0.16$ in both cases. Conversion efficiency for temperature independent case has the highest value for different material than for temperature dependent case. It is also noticeable that for temperature dependent case the relation between efficiency and hot side temperature in not linear. Maximum efficiency exceeded:

- $6,90 \%$ for $\mathrm{T}_{\mathrm{H}}=227^{\circ} \mathrm{C}, \mathrm{T}_{\mathrm{C}}=20^{\circ} \mathrm{C}, \mathrm{R}_{\mathrm{L}}=$ $0,00169 \Omega$ for $\mathrm{x}=0.16$ (constant thermoelectric parameters)

- $7,32 \%$ for $\mathrm{T}_{\mathrm{H}}=227^{\circ} \mathrm{C}, \mathrm{T}_{\mathrm{C}}=20^{\circ} \mathrm{C}, \mathrm{R}_{\mathrm{L}}=$ $0,00169 \Omega$ for $\mathrm{x}=0.26$ (temperature dependent thermoelectric parameters)

Nowadays, the numerical tools play an important role in development processes worldwide. The share of thermoelectric generators in the field of waste heat utilization increasing constantly. According to report [13] the market of thermoelectric generators will reach $\$ 875$ million by 2023 . Recent cutting edge solutions from the field of thermoelectric materials will contribute to the growth of more efficient and cheaper thermoelectric generators. Next steps in the research will cover:

- Investigations of performance parameters for thermocouple composed of p-type leg and ntype leg;

- Developing thermocouple model with soldering, contact layers, ceramics.

\section{Acknowledgments}

This work was supported by the funds of National Science Center granted on the basis of the decision No. DEC-2013/09/B/ST8/02043 and AGH grant No. 15.11.210.326 


\section{References}

1. H. Wei, Z. Gan, Z. Xingxing, J. Jie, L. Guiqiang, Z. Xudong, Appl. Energy 143, 1-25 (2015),

2. G. Schierning, R. Chaves, R. Schmechal, B. Balke, G. Rogl, P. Rogl, Transl. Mater. Res. 2 (2015),

3. D. Aswal, R. Basu, A. Singh, Energy Convers. Manage. 114, 50-67 (2016)

4. Z. Tian, S. Lee, G. Chen, Cond-mat.mtrl-sci (2014), 5. T. Caillat, J. Fleurial, G. Snyder, A. Borshchevsky, online: http://thermoelectrics.caltech.edu/SegmICT01.pdf (2001)

6. K. Wojciechowski, J. Merkisz, P. Fuć, J.

Tomankiewicz, R. Zybała, J. Leszczyński, P. Lijewski, P. Nieroda, CE 154, 60-71 (2013)

7. L. Weishu, J. Qing, K. Hee Seok, R. Zhifeng, Acta Mater. 87, 357-376 (2015)

8.T. Hua, J. Na, J. Qi, S. Xiuxiu, S. Gequn, L. Xingyu, Energy Procedia 75, 590-596(2015)

9. D. Crane, J. Electron. Mater. 40, 561-569(2010)

10. H. Kim, W. Kim, Appl. Energy 139, 205-211 (2015)

11. M. Picard, S. Turenne, D. Vasilevskiy, R. Masut, J. Electron. Mater. 42, 2343-2349(2013)

12. J. Jun, C. Lidong, B. Shengqiang, Y. Qin, W. Qun, J. Cryst. Growth 277, 258-263 (2005)

13. H. Zervos, Thermoelectric Energy Harvesting 20162025, 2016

14. H. Lee, Thermal Design, 2010

15. X. Zheng, C. Liu, Y. Yan, Q. Wang, Renew.

Sustainable Energy Rev. 32, 486-503 (2014)

16. E. Antonova, D. Looman, IEEE, 215-218 (2005)

17. S. Yongming, Z. Zhixiang, D. Yuan, Z. Wei, C. Xin, Z. Yongsheng, Energy Convers. Manage. 101, 713720 (2015)

18. N. Espinosa, M. Lazard, L. Aixala, H. Scherrer, J. Electron. Mater. 39, 1446-1455 (2010) 